\title{
Digital Elevation Model Production Using Point Cloud Acquired by Unmanned Aerial Vehicles
}

\author{
Suk Bae Lee, ${ }^{1}$ Jae Ho Won, ${ }^{2 *}$ Kap Yong Jung, ${ }^{3}$ \\ Mihwa Song, ${ }^{4}$ and Young Joon $\mathrm{Ahn}^{5}$ \\ ${ }^{1}$ Department of Civil Engineering, Gyeongnam National University of Science and Technology, \\ 33 Dongjin-ro, Jinju, Gyeongsangnam-do 52725, Republic of Korea \\ ${ }^{2}$ Department of Urban Engineering, Wonkwang University, \\ 460 Iksandae-ro, Iksan, Jeonbuk-do 54538, Republic of Korea \\ ${ }^{3}$ Department of Civil Engineering, Chungnam National University, \\ 99, Daehak-ro, Yuseong-gu, Daejeon 34134, Republic of Korea \\ ${ }^{4}$ ICT Convergence Research Division, Korea Expressway Corporation Research Institute, 208-96, Dongbu-daero \\ 922 beon-gil, Dongtan-myeon, Hwaseong-si, Gyeonggi-do 18489, Republic of Korea \\ ${ }^{5}$ Department of Geospatial Imagery and Photogrammetry, National Geographic Information Institute, \\ 92 Worldcup-ro, Yeongtong-gu, Suwon, Gyeonggi-do 16517, Republic of Korea
}

(Received July 1, 2020; accepted November 17, 2020)

Keywords: UAV, point cloud, DSM, DEM

Currently, point cloud data acquired by using unmanned aerial vehicles (UAVs) are mostly used for the production of digital surface models (DSMs). This paper shows the possibility of digital elevation model (DEM) production with point cloud data acquired using UAVs. In this study, which was conducted in Korea, we used 314 images acquired with a DJI Inspire-2 UAV. To extract ground data, we performed point cloud auto-classification with six types of software, Pix4DMapper, GlobalMapper, Inpho, Trimble Business Center (TBC), Metashape, and Terrascan, and the obtained results were compared. Pix4DMapper was used for point cloud extraction, and all six types of software used the default options for point cloud autoclassification. A point cloud acquired using LiDAR classifies vegetation using an echo, but a point cloud extracted from an image has no echo, making it impossible to classify vegetation using an echo. In this study, DEMs were produced using ground data classified automatically with the six types of software, and they were compared with a DEM produced by the Korean National Geographic Information Institute (NGII). As a result, the DEM error rate was 38$47 \%$ depending on the type of software used. In a mountainous area, the dense forest made it impossible to extract ground data, resulting in a very high error rate of $82-92 \%$.

\section{Introduction}

Recently, studies have been conducted to monitor and build databases for the ground surface environment, which is rapidly changing owing to the impact of urban development and climate change, in real time. ${ }^{(1,2)}$ Until now, most ground surface environmental monitoring to investigate climate and vegetation changes has been conducted using satellite images and aerial

*Corresponding author: e-mail: jhwon@nate.com

https://doi.org/10.18494/SAM.2020.2973 
photos. ${ }^{(3,4)}$ However, satellite images have a limitation in the shooting time due to the orbital period, while aerial photogrammetry has the disadvantage of being economically inefficient for use in small areas.

Structure from Motion (SfM), an image processing algorithm, is rapidly becoming widespread owing to its advantages of economic feasibility and efficiency in fields of applied surveying such as traditional civil engineering and architecture, and studies have been conducted to classify digital terrain models (DTMs) by analyzing 3D point clouds obtained from unmanned aerial vehicles (UAVs). ${ }^{(5)}$ Such point clouds have been studied to evaluate greenhouse gas emissions in large-scale forest developments and have also been used in the surveying field. ${ }^{(6)}$ The use of image sensors mounted on UAVs is expanding their application to various fields.

UAVs are an economical and efficient means of creating digital maps of small areas and building digital twins, because they can obtain high-resolution images more rapidly, more conveniently, and more economically than traditional aerial photogrammetry. ${ }^{(7-10)}$

At present, most of the images acquired using UAVs are used for the production of orthoimages and digital surface models (DSMs). In order to accurately calculate the volume of earth at a construction site, a digital elevation model (DEM), not a DSM, should be used.

In this study, a point cloud acquired using a UAV was automatically classified using six types of software [Pix4DMapper, GlobalMapper, Inpho, Trimble Business Center (TBC), Metashape, and Terrascan] and DEMs were produced. The purpose of this study is to compare the DEM and auto-classification results for the six types of software and to examine whether a DEM can be produced using a point cloud acquired with a UAV. To this end, a study area was selected and UAV photogrammetry was performed. The overall workflow is shown in Fig. 1.

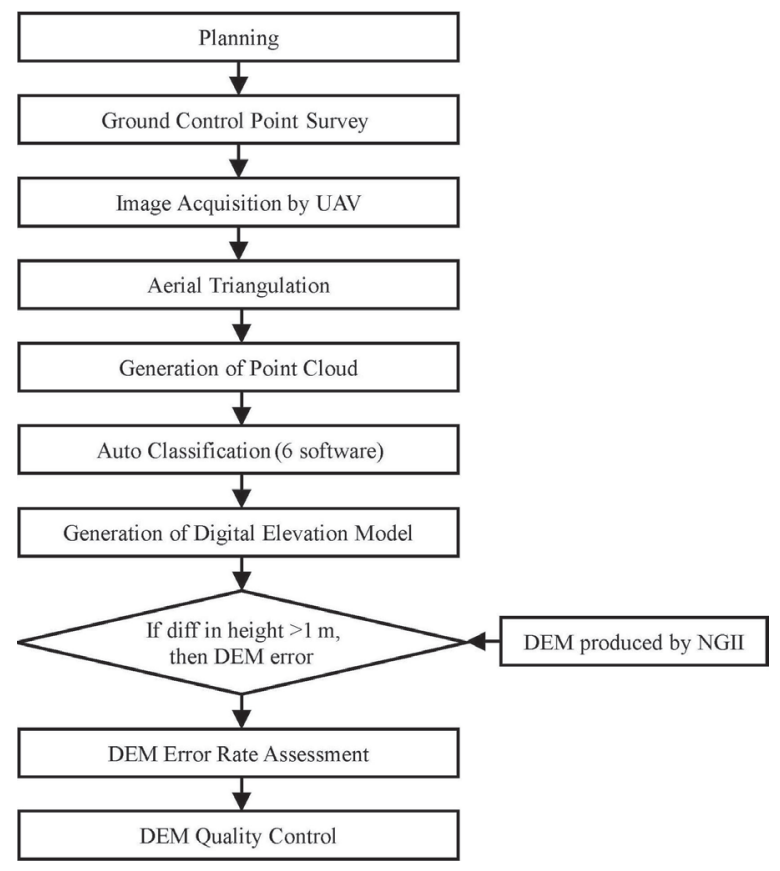

Fig. 1. Overall workflow adopted in this study. 
We selected a study area containing various topographic features such as downtown, roads, rivers, and mountains. As shown in Fig. 1, a work plan was first established for the study area, and then a ground control point (GCP) survey was conducted. Then, images of the study area were acquired using a DJI Inspire-2 UAV equipped with a DJI X5s camera, and aerial triangulation (AT) was performed. For point cloud extraction, commercial software (Pix4DMapper by Pix4D) was used, and auto-classification was performed using the six types of software. Finally, a comparison and an evaluation were carried out to distinguish the differences between the DEMs produced with the six types of software.

\section{Materials and Methods}

In Korea, in order to encourage public surveys using UAVs, the Korean National Geographic Information Institute (NGII) published "Guidelines for Public Survey Using UAV" (NGII Notice No. 2018-1075) in March 2018. ${ }^{(11)}$ Since these guidelines specify the standard working process and inspection standards, they should be followed when making digital maps using UAVs.

\subsection{Planning and GCP survey}

A planning stage was necessary to select the UAV, software, and study area, and to determine the ground sample distance (GSD), flight altitude, overlapping ratio, and so forth. In this study, a study area containing various topographic features was selected to compare and analyze the results of point cloud auto-classification for various topographic features. As shown in Fig. 2, the Namgang area in Jinju City was selected as the study area, which had an area of about $1.4 \mathrm{~km}^{2}$.

Article 9 of the NGII work guidelines stipulates that the GCPs should be distributed evenly in consideration of the shape of the work area and the direction of the shooting course, and there should be at least nine GCPs per $1 \mathrm{~km}^{2}$. $^{(1)}$ In addition, in accordance with Article 10 of the work guidelines, a horizontal control survey was performed by a GNSS network-RTK survey, while a vertical control survey was performed by a level survey. As a result of the ground

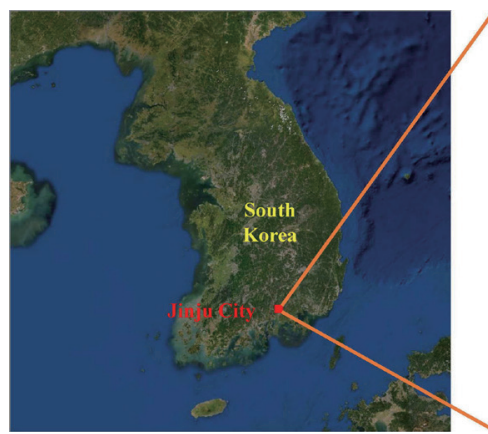

(a)

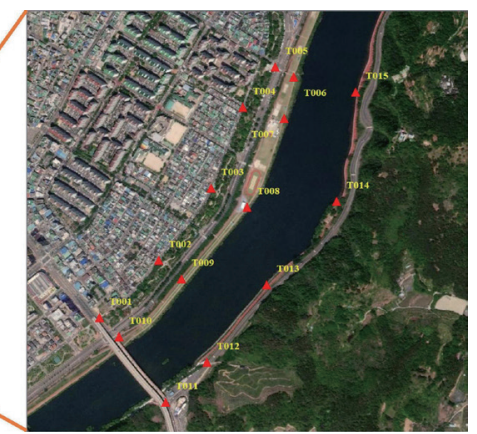

(b)

Fig. 2. (Color online) Photographs showing locations of (a) study area and (b) GCPs. 
control survey, transverse Mercator (TM) plane coordinates and heights were obtained for 15 GCPs. Figure 3(b) shows a photograph of the GNSS network-RTK survey.

\subsection{Image acquisition and AT}

In August 2019, a total of 342 images were acquired using a DJI Inspire-2 UAV. The planned flight altitude was $250 \mathrm{~m}$, the GSD was $5 \mathrm{~cm}$, the forward overlap was $80 \%$, and the side overlap was $60 \%$. A DJI X5s camera was used for image acquisition. Figure 4 shows the DJI Inspire-2 UAV and DJI X5s camera used for image acquisition. The camera specifications are shown in Table 1.

AT was performed with software that supports automatic tie-point matching and bundle block adjustment (BBA) in accordance with Article 17 of the NGII work guidelines. ${ }^{(11)}$ AT performs least-squares block adjustment to calculate the interior orientation (IO) and exterior

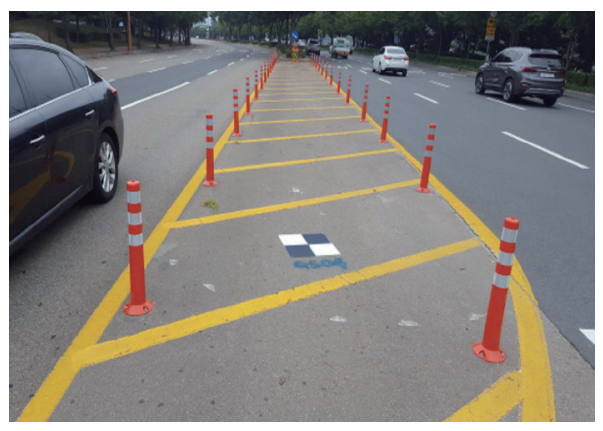

(a)

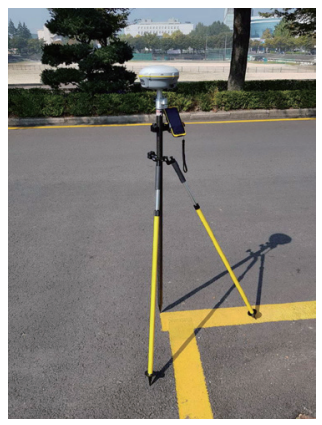

(b)

Fig. 3. (Color online) Photographs of (a) air photo signal and (b) horizontal GNSS network-RTK survey.

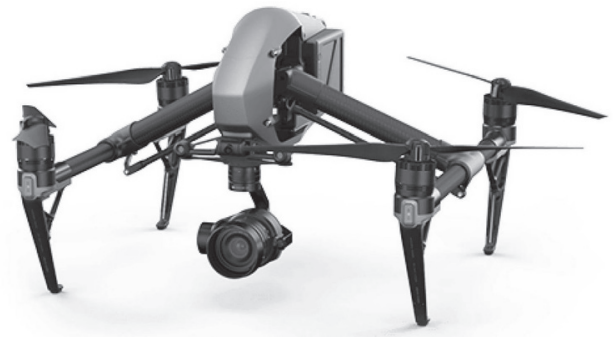

(a)

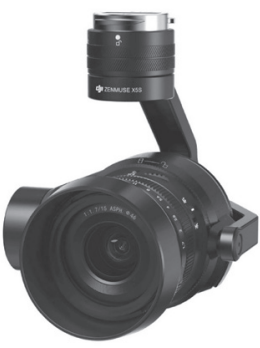

(b)

Fig. 4. (Color online) Photographs of (a) DJI Inspire-2 UAV and (b) DJI X5s camera.

Table 1

DJI X5s camera specifications.

\begin{tabular}{lc}
\hline Focal length & $15 \mathrm{~mm}$ \\
FOV & $72 \mathrm{deg}$ \\
Resolution & $5280 \times 3956(20.8 \mathrm{MP})$ \\
Weight & $461 \mathrm{~g}$ \\
Shutter speed & $8-1 / 8000 \mathrm{~s}$ \\
\hline
\end{tabular}


orientation (EO) parameters of the camera. The camera's IO parameters include the focal length, principal point, and lens distortion. The mathematical relationship between the image, camera geometry, and ground space is calculated via BBA, which is a strategy that minimizes errors. ${ }^{(12)}$ In this study, 15 GCPs and Pix4DMapper were used to perform the AT.

\subsection{Generation of point cloud and DSM}

Pix4DMapper was used to create the point cloud, and the data processing process is shown in Fig. 5. The process is as follows: (1) add aerial photos or a directory acquired by the UAV, (2) generate tie points on the images (optimize), (3) input GCP coordinates and mark GCP locations on photos, (4) reoptimize, (5) generate a point cloud, and (6) generate a DSM. Figures 6(a) and 6(b) respectively show the point cloud and DSM for the study area. The number of points in the point clouds in the study area was 46371790 and the point density was $34 \mathrm{pt} / \mathrm{m}^{2}$.

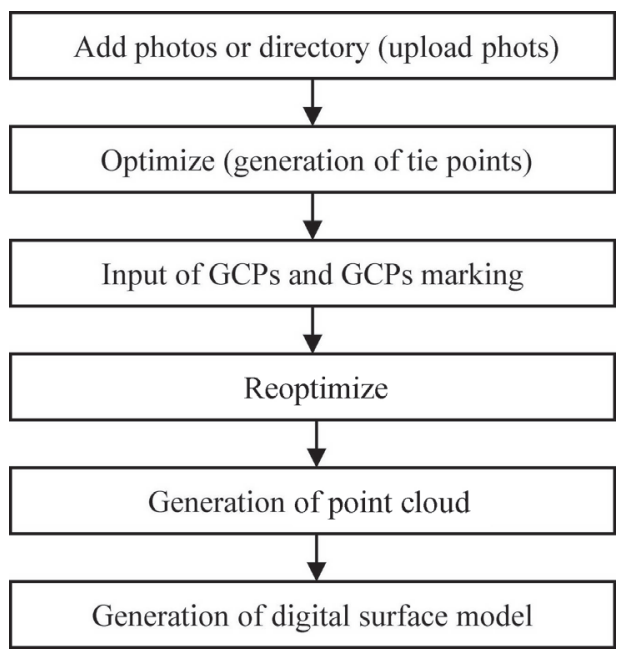

Fig. 5. Flowchart of data processing using Pix4DMapper.

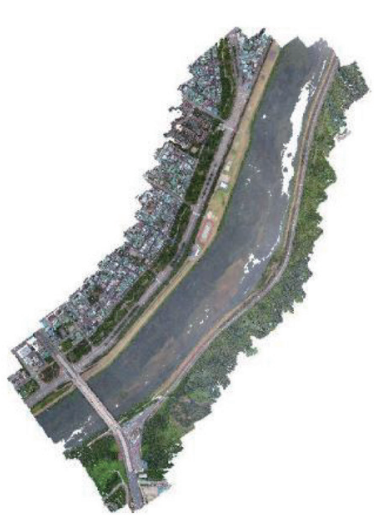

(a)

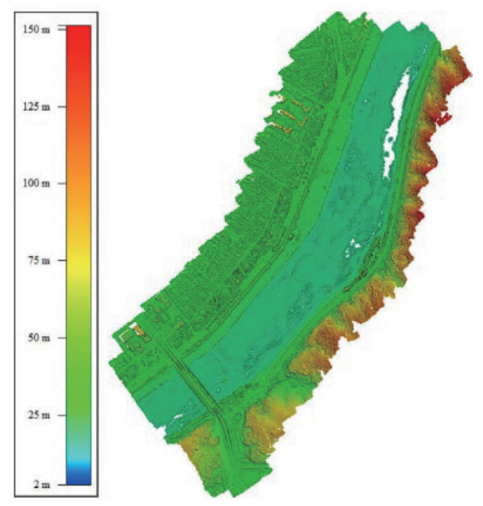

(b)

Fig. 6. (Color online) (a) Point cloud and (b) DSM of study area. 


\subsection{Auto-classification and generation of DEM}

In this study, the point cloud was automatically classified with six types of software, Pix4D's Pix4DMapper, Blue Marble Geographic's GlobalMapper, Trimble's Inpho and TBC, Agisoft's Metashape, and Terrasolid's Terrascan, DEMs were generated, and the results were compared. All six types of software performed auto-classification using the default options.

Pix4DMapper auto-classifies areas into five items: ground, road surfaces, high-density vegetation, buildings, and manmade objects, and there are no detailed options for autoclassification that users can select. The auto-classification results of Pix4DMapper in Fig. 7 show that there is little ground data for the mountainous area, and some buildings are included in the ground data.

Auto-classification using GlobalMapper provides various options, as shown in Fig. 8, and it can automatically classify a point cloud into ground, buildings, high-density vegetation, and powerlines. According to the auto-classification results in Fig. 9, the high-density vegetation area consists mostly of buildings, with some of them classified into ground, and the ground contains many buildings.

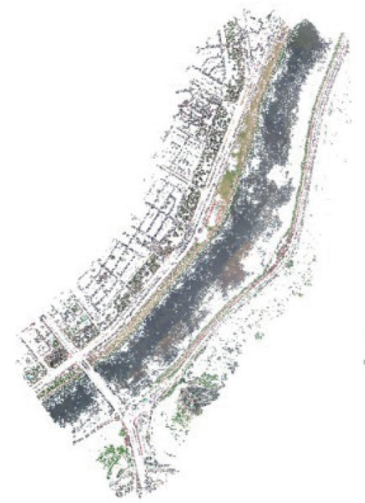

(a)

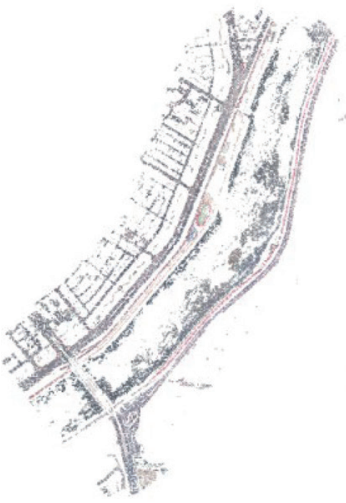

(b)

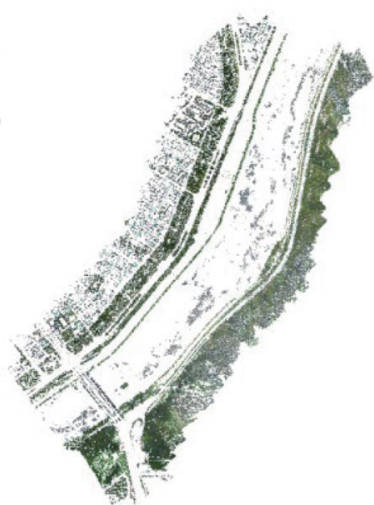

(c)

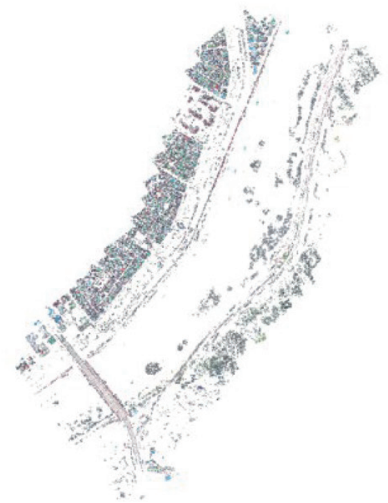

(d)

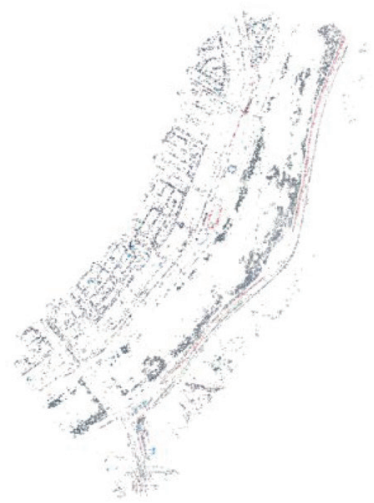

(e)

Fig. 7. (Color online) (a) Ground, (b) road surfaces, (c) high-density vegetation, (d) buildings, and (e) manmade objects classified using Pix4DMapper. 


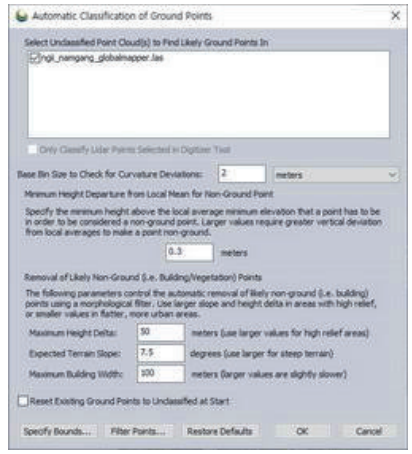

(a)

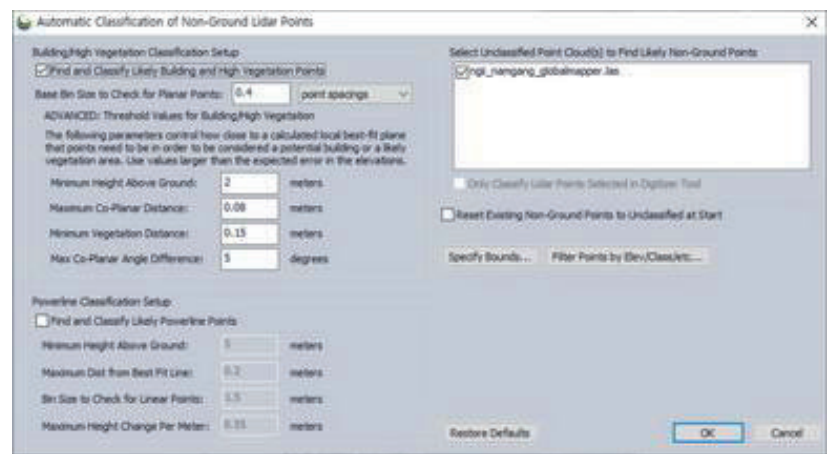

(b)

Fig. 8. (Color online) Screenshots of options when auto-classifying (a) ground and (b) non-ground using GlobalMapper.

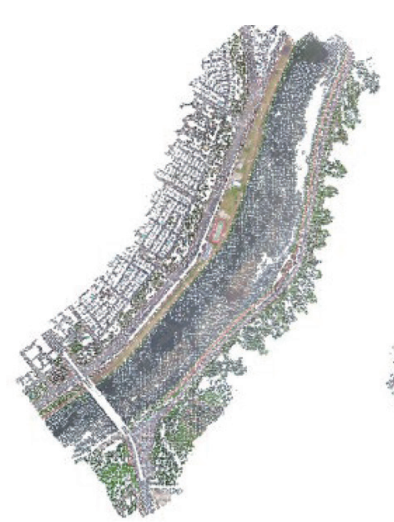

(a)

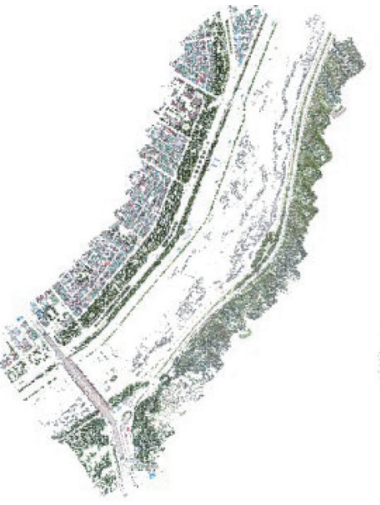

(b)

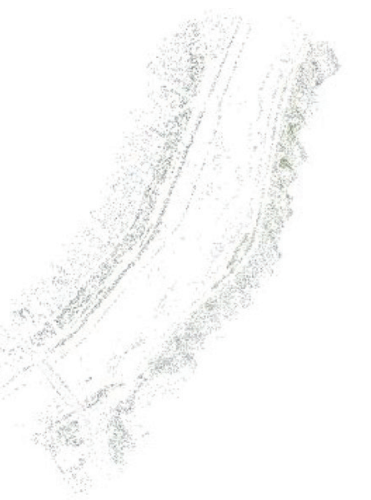

(c)

Fig. 9. (Color online) (a) Ground, (b) buildings, and (c) high-density vegetation classified using GlobalMapper.

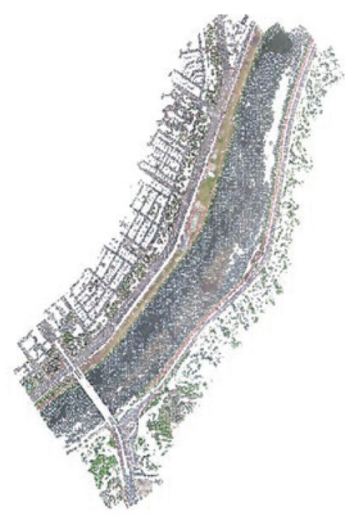

(a)

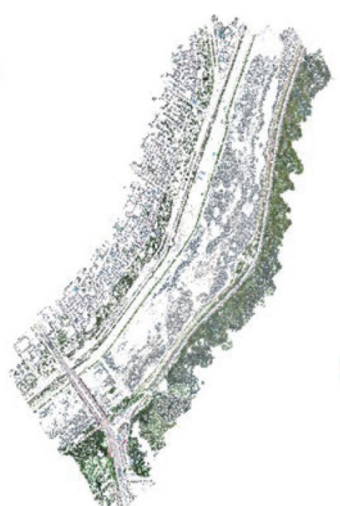

(b)

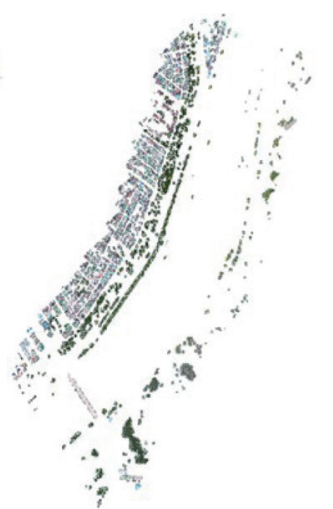

(c)

Fig. 10. (Color online) (a) Ground, (b) high-density vegetation, and (c) buildings classified using Inpho.

Inpho uses the DTM toolkit to perform auto-classification and there are no detailed options. Auto-classification involves three categories: ground, high-density vegetation, and buildings. According to the auto-classification results in Fig. 10, some buildings and high-density vegetation were classified as ground. 
TBC auto-classifies areas into buildings, ground, high-density vegetation, poles and signs, and powerlines, and there are no detailed options for auto-classification. Figure 11 shows that most buildings were classified into high-density vegetation, while most of the mountainous area was classified into high-density vegetation, resulting in almost no ground data. In addition, some buildings and bridges were classified into ground.

Metashape auto-classifies areas into ground, high-density vegetation, buildings, road surfaces, cars, and manmade objects, and there are no detailed options. According to the results of Metashape auto-classification in Fig. 12, a large proportion of high-density vegetation and buildings were classified into ground.

As shown in Fig. 13, Terrascan provides various auto-classification options and functions. According to the results of auto-classification in Fig. 14, some of the high-density vegetation was classified into ground, and most of the high-density vegetation was classified into buildings.

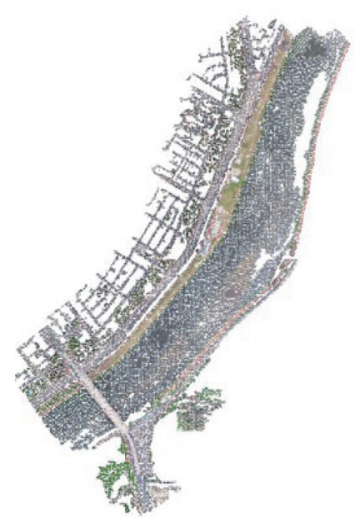

(a)

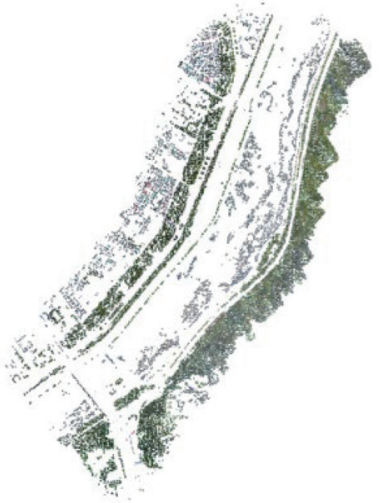

(b)

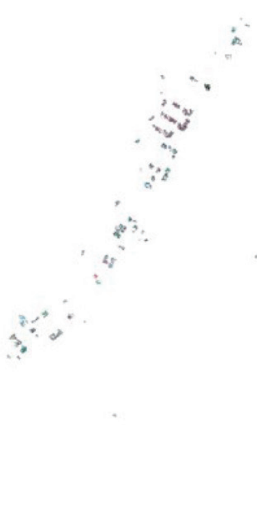

(c)

Fig. 11. (Color online) (a) Ground, (b) high-density vegetation, and (c) building areas classified using TBC.

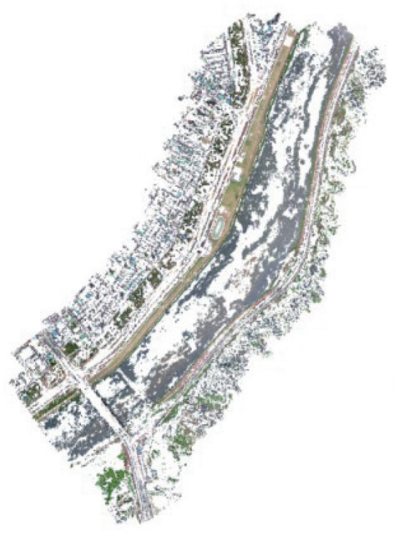

(a)

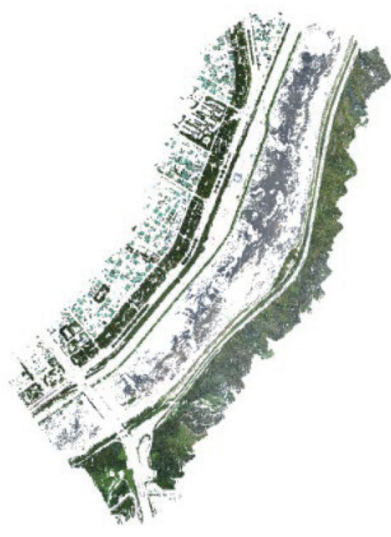

(b)

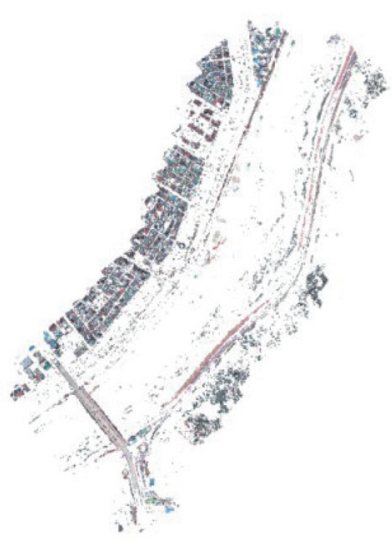

(c)

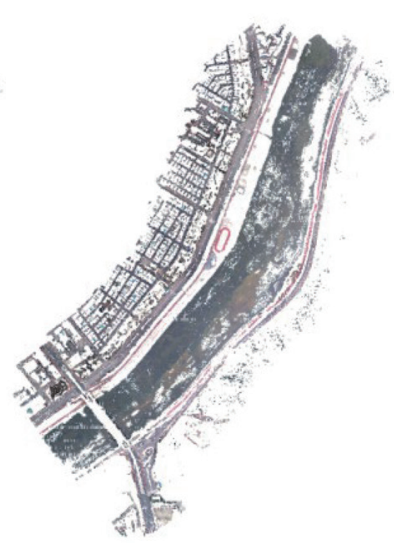

(d)

Fig. 12. (Color online) (a) Ground, (b) high-density vegetation, (c) buildings, and (d) road surfaces classified using Metashape. 


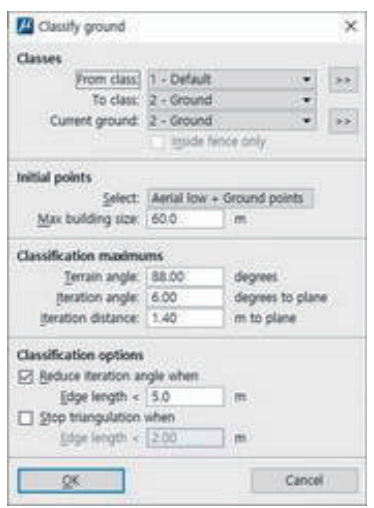

(a)

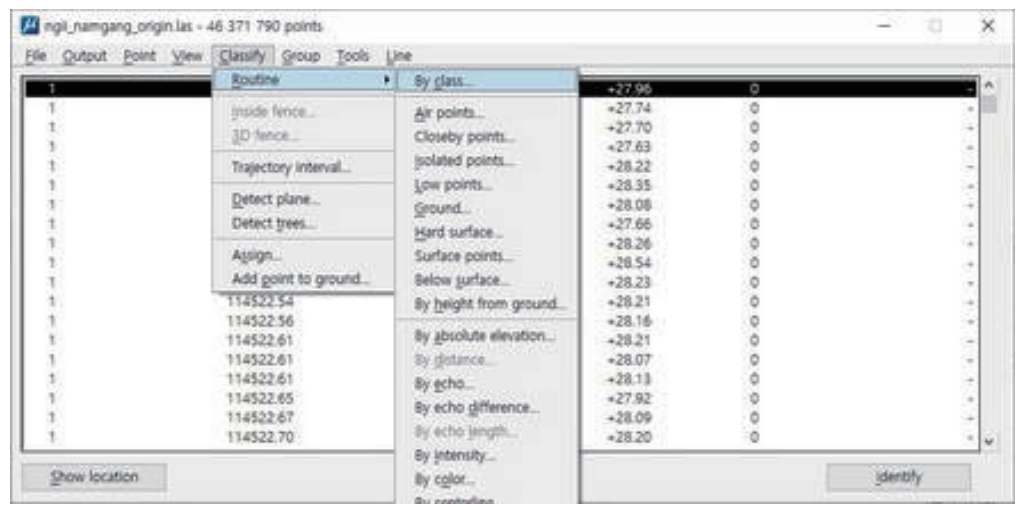

(b)

Fig. 13. (Color online) Screenshots of (a) ground classification option and (b) classification function using Terrascan.

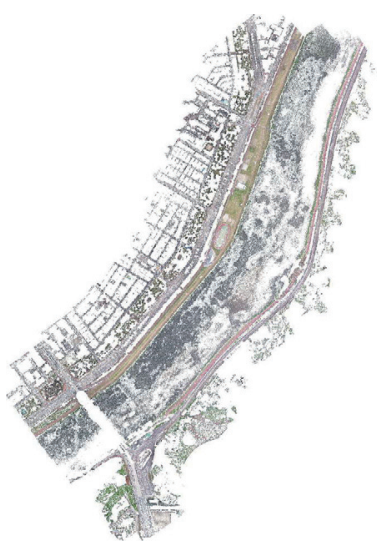

(a)

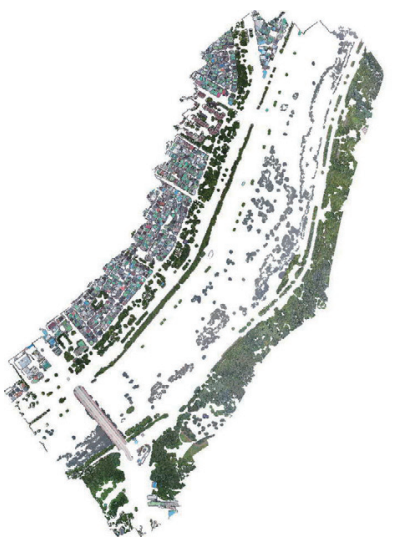

(b)

Fig. 14. (Color online) (a) Ground and (b) building classified using Terrascan.

After automatically classifying the point cloud using the six types of software, we generated DEMs using the auto-classified ground data. Figure 15 shows the DEMs for the six types of software.

\subsection{DEM quality control}

For the DEM quality control, AT and DEM accuracy assessments were carried out. For AT accuracy assessment, the root mean square error (RMSE) and maximum error of the GCPs were calculated. The RMSE of the GCPs was calculated using the residuals between the GCP coordinates acquired through the GCP survey and the GCP coordinates calculated by performing AT in Pix4DMapper. The results can be seen through the Pix4DMapper quality report and are shown in Table 2. The RMSEs in the $X, Y$, and $Z$ directions are 2, 3.7, and $0.1 \mathrm{~cm}$, and the maximum errors are 4.6, 6.2, and $2.1 \mathrm{~cm}$, respectively. In Korea, the plane position and 


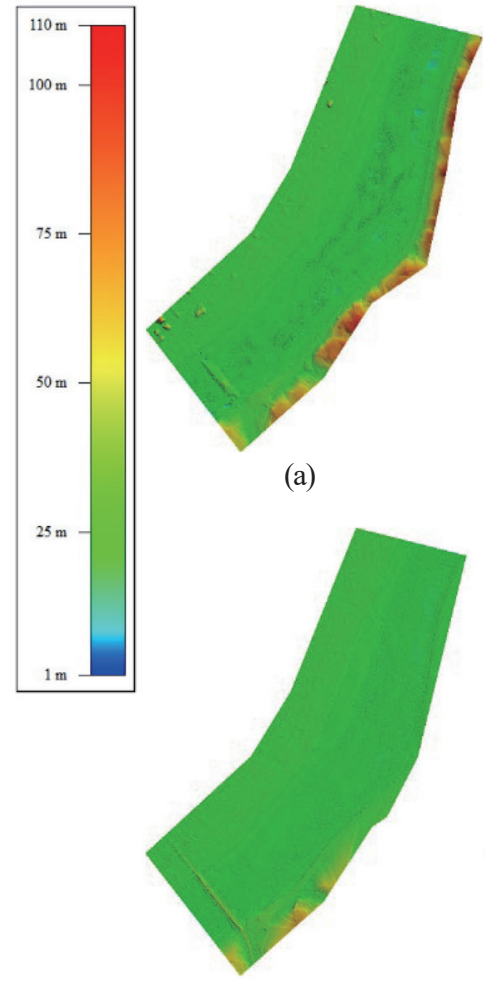

(d)

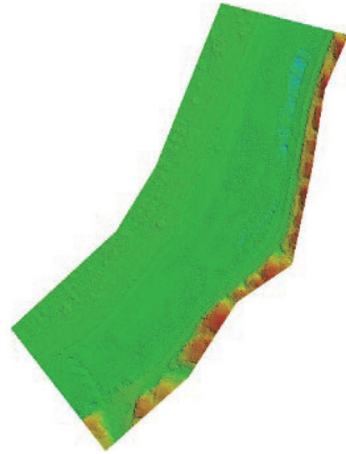

(b)

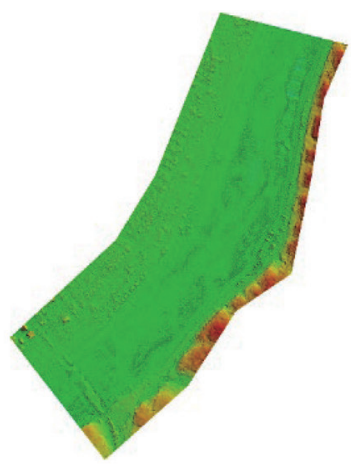

(e)

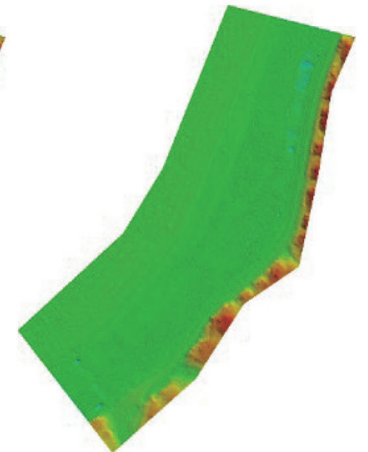

(c)

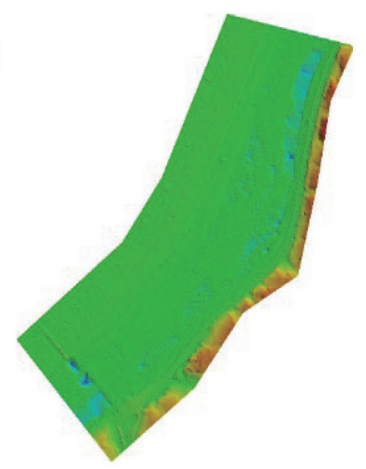

(f)

Fig. 15. (Color online) Generated DEM using ground data classified by (a) Pix4DMapper, (b) GlobalMapper, (c) Inpho, (d) TBC, (e) Metashape, and (f) Terrascan.

Table 2

RMSE and maximum error of GCPs in AT (unit: $\mathrm{cm}$ ).

\begin{tabular}{llll}
\hline Division & $\Delta X$ & $\Delta Y$ & $\Delta Z$ \\
\hline RMSE & 2.0 & 3.7 & 1.0 \\
Maximum error & 4.6 & 6.2 & 2.1 \\
\hline
\end{tabular}

height accuracy are supposed to follow the Aerial Photogrammetry Regulations. ${ }^{(13)}$ According to Article 56 of the Aerial Photogrammetry Regulations, the limits of adjustment calculations and errors are defined as $0.2 \mathrm{~m}$ (RMSE) and $0.4 \mathrm{~m}$ (maximum error) for scales from 1:1000 to 1:1200. The AT result meets this Korean standard, which means that Pix4DMapper performed AT well.

For the DEM accuracy assessment, the difference in height from the DEM of the NGII in Fig. 16 with a grid size of $5 \mathrm{~m}$ was calculated, as shown in Fig. 17, and a height difference of $1 \mathrm{~m}$ or more was determined as a DEM error. The DEM error rate for the six types of software was $38-47 \%$, and the DEM error rates according to the terrain were $50-63 \%$ in the river area, $82-92 \%$ in the mountainous area, and $15-28 \%$ in the urban area. 


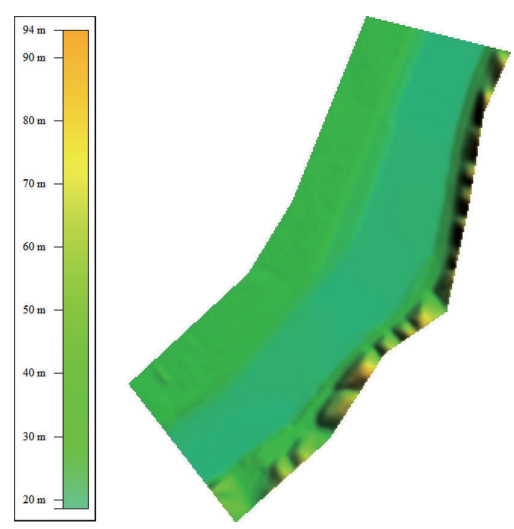

Fig. 16. (Color online) DEM produced by Korean NGII.

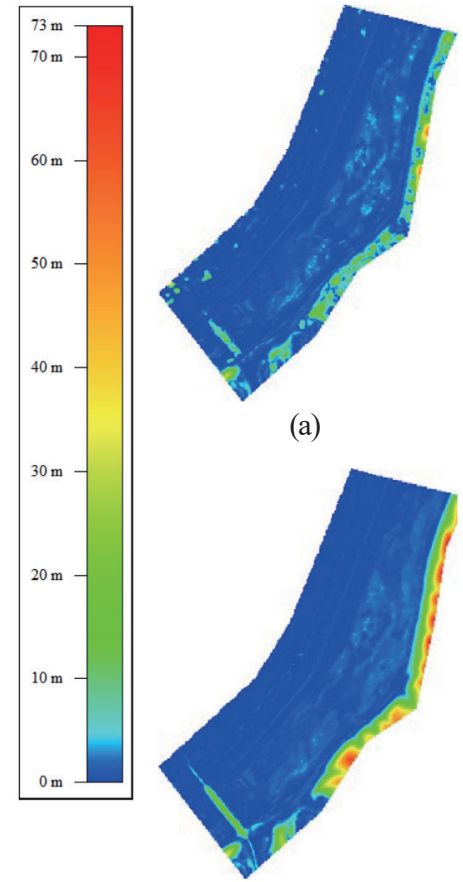

(d)

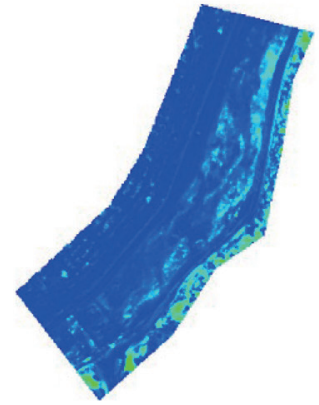

(b)

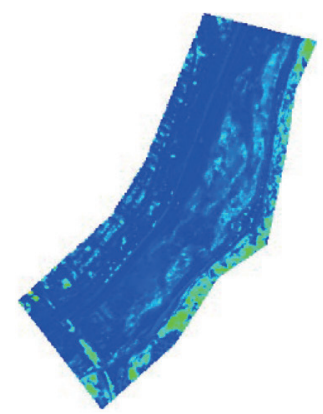

(e)

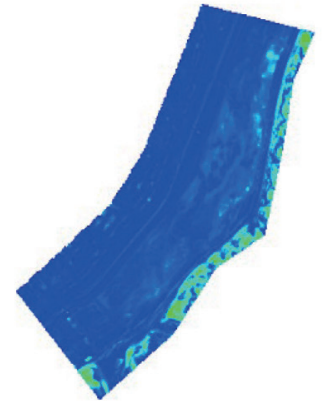

(c)

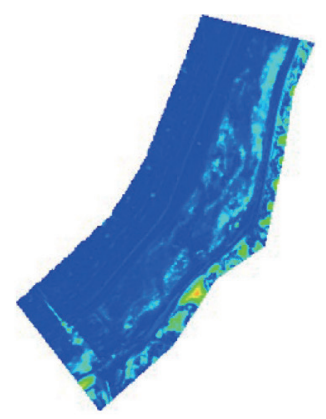

(f)

Fig. 17. (Color online) DEM height differences of (a) Pix4DMapper, (b) GlobalMapper, (c) Inpho, (d) TBC, (e) Metashape, and (f) Terrascan.

\section{Results and Discussion}

In this study, UAV photogrammetry, point cloud auto-classification, and DEM generation were performed. The accuracy of AT, the most important process of UAV photogrammetry, was found to be excellent. The point cloud auto-classification was performed for the six types of software and DEMs were produced, then the DEM accuracy was evaluated by calculating the difference in height from the DEM of the NGII. The DEM error rate was calculated for sampled (grid center) points. Table 3 shows the DEM error rates for the six types of software. 
Table 3

Error rates of DEMs.

\begin{tabular}{lcccccc}
\hline Area & Pix4DMapper & Inpho & Metashape & TBC & GlobalMapper & Terrascan \\
\hline River area & $50 \%$ & $53 \%$ & $61 \%$ & $58 \%$ & $63 \%$ & $63 \%$ \\
Mountainous area & $87 \%$ & $84 \%$ & $84 \%$ & $92 \%$ & $82 \%$ & $82 \%$ \\
Urban area & $16 \%$ & $15 \%$ & $28 \%$ & $17 \%$ & $20 \%$ & $17 \%$ \\
Total & $38 \%$ & $38 \%$ & $47 \%$ & $42 \%$ & $44 \%$ & $42 \%$ \\
\hline
\end{tabular}

The terrain of the study site comprised 37\% river area, 13\% mountainous area, and 50\% urban area. In the mountainous area, the dense forest made it impossible to extract ground data, which led to the high DEM error rate of $82-92 \%$. In the urban area, the DEM error rate was relatively low (15-28\%), and the main cause of the DEM error was that buildings and street trees to be removed when extracting the ground data were included in the ground data.

\section{Conclusions}

The purpose of this study was to investigate the possibility of DEM production based on the images acquired using a UAV. The UAV photogrammetry involved planning, GCP survey, image acquisition, and AT in this order, and the overall order of DEM production was point cloud extraction, auto-classification, DEM generation, and DEM quality control. The study was conducted in Korea, and 342 images were acquired using a DJI Inspire-2 UAV and a DJI $\mathrm{X} 5 \mathrm{~s}$ camera. UAV photogrammetry was performed in accordance with the Guidelines for the Public Survey Using UAV, which defines the standard work process and inspection standards in Korea. In this study, automatic point cloud classification was performed and DEMs were produced using six types of software: Pix4DMapper, GlobalMapper, Inpho, TBC, Metashape, and Terrascan. The DEMs were compared with that of the NGII, and the DEM error rate was $38-47 \%$ but was as high as $82-92 \%$ in the densely forested mountainous area.

In order to produce an accurate DEM, it is important to accurately classify the ground in point clouds. Currently, manual classification is performed because point clouds cannot be classified accurately only with auto-classification. Therefore, in order to activate DEM production using UAVs, the development of point cloud auto-classification technology and the introduction and testing of LiDAR are necessary for mountainous areas with dense forest.

\section{Acknowledgments}

This work was supported by the National Geographical Information Institute in Korea as a 2019 research project with the name "A Study on Policy Development to Promote the Utilization of Unmanned Air Vehicle Space Information" and the Korea Expressway Corporation Research Institute as a 2019-2020 research project with the name "A Study on the Plan Establishment of Standard Work and Pilot Operation for Use of Drones in Construction Field". 


\section{References}

1 D. I. Kim, Y. S. Song, K. H. Kim, and C. W. Kim: J. Korean Soc. Geospatial Inf. Sci. 32 (2014) 29. https://doi. org/10.7848/ksgpc.2014.32.1.29

2 B. G. Lee: J. Korean Earth Sci. Soc. 39 (2018) 46. https://doi.org/10.5467/JKESS.2018.39.1.46.

3 D. X. Tran, F. Pla, P. L. Carmona, S. W. Myint, M. Caetano, and H. V. Kieu: ISPRS J. Photogram. Remote Sens. 124 (2017) 119. https://doi.org/10.1016/j.isprsjprs.2017.01.001.

4 J. Kim and E. J. Kim: J. Korean Soc. Surv. Geodesy Photogram. Cartography 37 (2019) 143. https://doi. org/10.7848/ksgpc.2019.37.3.143.

5 N. Polata and M. Uysal: ISPRS XLII-4/W6 (2017). https://doi.org/10.5194/isprs-archives-XLII-4-W6-77-2017.

6 R. Mlambo, I. H. Woodhouse, F. Gerard, and K. Anderson: Forests 8 (2017) 68. https://doi.org/10.3390/ f8030068

7 M. J. Ahmad, A. Ahmad, and K. D. Kanniah: 2018 IOP Conf. Ser.: Earth Environ. Sci. 169 (2018) 012077.

8 X. Tianyun, T. Xiaocheng, Y. Defang, X. Yonghe, and Y. Hongliang: Int. J. Control Autom. 8 (2015) 279. https://doi.org/10.14257/ijca.2015.8.3.28

9 I. Colomina and P. Molina: ISPRS J. Photogram. Remote Sens. 92 (2014) 79. https://doi.org/10.1016/ j.isprsjprs.2014.02.01

10 A. Ahmad: Pertanika J. Sci. Technol. 19(S) (2011) 51

11 National Geographical Information Institute: Guidelines for the Public Survey Using UAV. NGII Notice No. 2018-1075 (2018)

12 M. Koeva, M. Muneza, C. Gevaert, M. Gerke, and F. Nex: Surv. Rev. 50 (2018) 361. https://doi:10.1080/003962 65.2016.1268756

13 National Geographical Information Institute: Aerial Photogrammetry Regulations. NGII Notice No. 20162609 (2016).

\section{About the Authors}

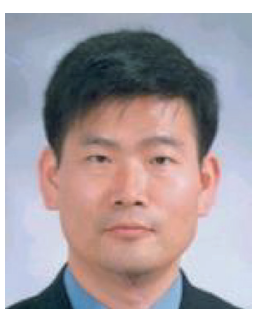

Suk Bae Lee received his B.E., M.S., and Ph.D. degrees from Sungkyunkwan University, Korea, in 1986, 1989, and 1997, respectively. From 1997 to 2003, he worked as a professor at Vision College of Jeonju. Since 2004, he has been a professor at Gyeongnam National University of Science and Technology, Korea. He was the president of the Korean Society for Geospatial Information Science in 2018. His research interests are in geoid modeling, GNSS application, and UAV photogrammetry. (sblee@gntech.ac.kr)

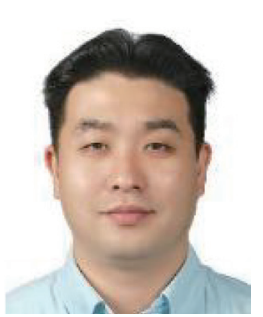

Jae Ho Won received his B.E. degree from Korea Advanced Institute of Science and Technology (KAIST), Korea, in 1996, his M.S. degree from Seoul National University, Korea, in 1998, and his Ph.D. degree from Chungnam National University, Korea, in 2013. Since 2018, he has been a research professor at Wonkwang University, Korea. His research interests are in UAV photogrammetry, camera calibration, and remote sensing. (aerowon@nate.com)

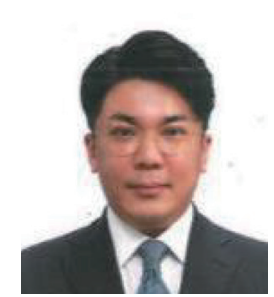

Kap Yong Jung received his B.S., M.S., and Ph.D. degrees in civil engineering from Chungnam National University, Korea, in 2003, 2009, and 2013, respectively. Since 2019, he has been a visiting professor at Chungnam National University, Korea. His research interests are in the areas of geodetic science, surveying, and geospatial information. (jungjusa@cnu.ac.kr) 


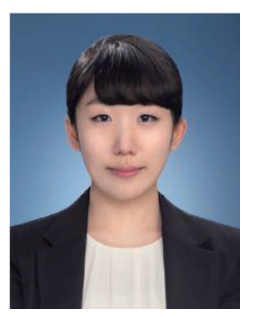

Mihwa Song received her B.S. degree from the Department of Radio Communication Engineering, Korea Maritime and Ocean University, Busan, Korea, in 2011 and her Ph.D. degree from the Department of Electrical and Computer Engineering, the University of Seoul, Seoul, Korea, in 2015. She is currently working as a deputy principal researcher at Korea Expressway Corporation Research Institute, Hwaseong, South Korea. Her current research interests include wireless communications, cooperative communications, cognitive radio systems, digital multimedia broadcasting, cell broadcast services, and wireless emergency alert systems. (mihwa@ex.co.kr)

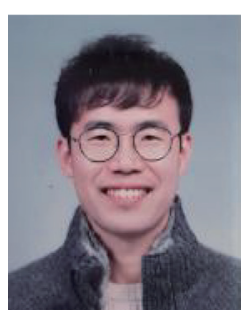

Young Joon Ahn received his B.S. and M.S. degrees from Sungkyunkwan University, Korea, in 1999 and 2002, respectively. He is currently a Ph.D. student at Sungkyunkwan University. Since 2006, he has been a public officer at NGII (National Geographical Information Institute), the Ministry of Land, Infrastructure and Transport, Korea. His research interests are in photogrammetry, geoid modeling, GNSS, and UAV surveying. (4mylove@korea.kr) 